\title{
Centros de Informação e Assistência Toxicológica no Brasil: descrição preliminar sobre sua organização e funções
}

\author{
Brazilian Poison Control Centers: preliminary description of their \\ organization and functions
}

Aline de Oliveira Costa', Herling Gregorio Aguilar Alonzo ${ }^{\mathbf{1}}$

DOI: $10.1590 / 0103-1104201912008$

RESUMO Esta pesquisa teve por objetivo descrever os atributos funcionais e organizacionais dos Centros de Informação e Assistência Toxicológica (CIATox) inseridos na Rede de Atenção as Urgências e Emergências do Sistema Único de Saúde (SUS). Realizou-se um estudo descritivo conduzido por meio de um questionário on-line, disponibilizado em sítio eletrônico e aplicado aos profissionais representantes dos CIATox. Dos 31 CIATox convidados, 19 (61\%) aceitaram e foram incluídos no estudo. Os CIATox participantes oferecem atendimento em tempo integral de forma telefônica e/ou presencial, e 39 tipos de atividades, com destaque para a assessoria clínica para profissionais de saúde. Estão vinculados a instituições gestoras nos três níveis de governo, contudo, possuem recursos limitados, sobretudo pelo financiamento inadequado ou insuficiente. Os CIATox podem contribuir com sua expertise para a visibilidade das intoxicações no Brasil e para o desenvolvimento de práticas de atenção à saúde da pessoa exposta ou intoxicada, integradas e operacionalizadas por meio de redes de atenção à saúde.

PALAVRAS-CHAVE Envenenamento. Centro de controle de intoxicações. Assistência à saúde. Sistema Único de Saúde.

\begin{abstract}
This study aimed to describe the functional and organizational attributes of Brazilian Poison Control Centers (PCC) inserted in the Urgency e Emergency Care Network of the Unified Health System (SUS). A descriptive study was conducted through an online questionnaire, made available on an electronic website and applied to professionals representing the PCC. Out of the 31 CIATox invited, 19 (61\%) accepted and were included in the study. PCC offer full-time telephone and/or face-to-face care and 39 types of activities, with emphasis on clinical advice for health professionals. They are linked to management institutions at all three levels of government, however, they have limited resources, mainly due to inadequate or insufficient funding. PCC can contribute with its expertise to the visibility of intoxications in Brazil and to the development of health care practices of the exposed or intoxicated person, integrated and operationalized through health care networks.
\end{abstract}




\section{Introdução}

As exposições a substâncias químicas e toxinas que resultam em intoxicações e envenenamentos afetam todos os gêneros e todas as idades, em condições não intencionais, tais como os acidentes domésticos, ocupacionais e ambientais; e em circunstâncias intencionais, como, por exemplo, as lesões autoinfligidas, suicídios, homicídios e agressões ${ }^{1}$.

Em âmbito mundial, a incidência de intoxicações é desconhecida. Em relação à mortalidade, contudo, estima-se que as intoxicações causadas por produtos químicos de origem industrial e agrícola são responsáveis por 1,2 milhão de mortes, $2 \%$ do total de óbitos no mundo ${ }^{2}$. Intoxicações não intencionais foram associadas a 193 mil mortes em $2012^{3}$ e a mais de 100 mil mortes em $2016^{4}$. Embora a mortalidade por intoxicações não intencionais tenha decrescido no período, as taxas continuam elevadas nos países em desenvolvimento ${ }^{5}$.

No Brasil, tanto as intoxicações exógenas como os acidentes por animais peçonhentos são considerados agravos de notificação compulsória e devem ser registrados no Sistema de Informação de Agravos de Notificação (Sinan) por meio do preenchimento de Ficha de Notificação e Investigação específica para cada caso ${ }^{6}$.

No período de 2010 a 2017, o Sinan registrou 703.928 casos de intoxicações, com 7.840 óbitos e 1.077.399 acidentes por animais peçonhentos, com 1.752 óbitos ${ }^{7}$. Contudo, a magnitude da morbimortalidade por intoxicações e envenenamentos pode ser muito maior, uma vez que foram constatadas subnotificações dos casos nesse sistema de informação ${ }^{8,9}$.

O reconhecimento das exposições e intoxicações como um problema de saúde pública favoreceu a criação de serviços especializados, denominados internacionalmente como Centros de Controle de Intoxicações, que têm por objetivo oferecer informações, assessorar a prevenção, diagnóstico, prognóstico e tratamento das intoxicações ${ }^{10}$.

Esses serviços foram criados no Brasil a partir da década de 1970, sob diferentes características e denominações ${ }^{11,12}$. Todavia, somente em 2015 o Ministério da Saúde (MS) instituiu 31 desses serviços como estabelecimentos de saúde integrantes da Linha de Cuidado ao Trauma (LCT), da Rede de Atenção as Urgências e Emergências (RUE) no Sistema Único de Saúde (SUS), sob a denominação de Centros de Informação e Assistência Toxicológica (CIATox) ${ }^{\mathbf{1 3}}$, definidos como

unidades de saúde, de referência em Toxicologia Clínica no SUS, com atendimento em regime de plantão permanente por teleconsultoria e/ou presencial, com o objetivo de prover informação toxicológica aos profissionais de saúde e às instituições e prestar assistências às pessoas expostas e/ou intoxicadas, visando à redução de morbimortalidade.

O MS estabeleceu, ainda, atividades essenciais e opcionais, e institui um incentivo financeiro para esses serviços ${ }^{13}$.

Embora a descrição do perfil epidemiológico dos atendimentos realizados por diferentes CIATox seja discutida na literatura nacional ${ }^{14-17}$, poucos estudos abordam seus aspectos organizacionais ${ }^{18,19}$; e não há estudos com tais explanações após a sua inserção no SUS. Dessa forma, este artigo tem por objetivo, portanto, descrever os atributos funcionais e organizacionais dos CIATox brasileiros.

\section{Métodos}

Trata-se de um estudo descritivo conduzido por meio de um questionário on-line, realizado com coordenadores ou responsáveis técnicos dos CIATox, durante o período de fevereiro a abril de 2018.

O questionário consistiu em um conjunto de questões quantitativas e qualitativas, que cobriram os atributos funcionais (meio de atendimento e atividades ofertadas) e os atributos organizacionais (vinculação institucional e gestão, recursos humanos, recursos materiais e infraestrutura e financiamento) 
dos CIATox. Foi confeccionado em plataforma web por um profissional desenvolvedor, que utilizou o sistema de código aberto de gestão de conteúdo $w e b^{\mathbf{2 0}}$ denominado Joomla! ${ }^{\mathrm{TM}}$, e disponibilizado em um sítio eletrônico de domínio particular, pago com recursos próprios dos pesquisadores.

A fim de verificar o método, a pertinência das questões e a usabilidade do sítio eletrônico, foi realizado um piloto do preenchimento do questionário com um dos CIATox, seguido das adequações constatadas como necessárias.

Para a pesquisa, foram considerados os 31 CIATox instituídos pelo MS como estabelecimentos de saúde integrantes da LCT da RUE, no âmbito do SUS ${ }^{13}$, incluindo o CIATox participante do piloto.

Os convites para participação na pesquisa, juntamente com as informações de acesso ao questionário, foram enviados por e-mail. Cada CIATox convidado recebeu um nome de usuário e uma senha exclusiva, gerada automaticamente pelo sistema. Para garantir a segurança da informação, nem o desenvolvedor nem os pesquisadores tiveram acesso às senhas dos usuários disponibilizadas pelo sistema.

O questionário on-line ficou disponível para os CIATox por um total de 30 dias. Durante esse período, foram realizados contatos telefônicos e envio de e-mail para os profissionais dos CIATox como maneira de acompanhamento. Ao final do período, as respostas recebidas foram transferidas para um banco de dados construído no programa Microsoft ${ }^{\circledR}$ Office Excel ${ }^{\circledR} 2007$, o qual também proporcionou a apresentação dos dados conforme a estatística descritiva, por meio do cálculo de medidas de tendência, frequências e proporções.

Este manuscrito é parte de uma pesquisa maior que tem por objetivo analisar as práticas dos serviços de saúde direcionadas à atenção à saúde das pessoas expostas ou intoxicadas e contribuir para a identificação e definição da LC na RAS do SUS, cujo projeto foi submetido para apreciação do Comitê de Ética em Pesquisa (CEP) da Universidade Estadual de Campinas (Unicamp), sendo aprovado por meio do parecer $\mathrm{n}^{\mathrm{o}} 2.450 .750$ em 20 de dezembro de 2017.

\section{Resultados}

Dos 31 CIATox convidados a participar da pesquisa, 19 (61\%) aceitaram e foram incluídos no estudo, sendo um deles participante do teste-piloto. Dos CIATox não participantes, quatro não acessaram o questionário; sete não responderam e um recusou a participação.

Os 19 CIATox participantes estão distribuídos em 12 estados, nas cinco regiões do Brasil, em maior número no estado de São Paulo (quadro 1). Os 12 CIATox não participantes estão localizados no Amazonas, no Ceará, no Espírito Santo, em Goiás, no Rio de Janeiro, no Rio Grande do Norte, em Sergipe, no Paraná (dois CIATox) e em São Paulo (três CIATox).

\begin{tabular}{llll}
\hline \multicolumn{2}{l}{ Quadro 1. Distribuição dos CIATox participantes segundo localização, por Região, Unidade Federativa e Município. Brasil, 2018} \\
\hline Região do Brasil & Unidade Federativa & Município & Nome do CIATox ${ }^{\star}$ \\
\hline Norte & Pará & Belém & Centro de Informações Toxicológicas de Belém \\
\hline Nordeste & Bahia & Salvador & Centro Antiveneno da Bahia \\
& Paraíba & Campina Grande & $\begin{array}{l}\text { Centro de Informações e Assistência Toxicológica de } \\
\text { Campina Grande }\end{array}$ \\
& João Pessoa & Centro de Assistência Toxicológica de João Pessoa \\
& Recife & Centro de Assistência Toxicológica de Pernambuco \\
& Piauí & Teresina & Centro de Informações Toxicológicas de Teresina \\
\hline
\end{tabular}




\begin{tabular}{llll}
\hline Quadro 1. (cont.) & & & \\
\hline Centro-Oeste & Distrito Federal & Brasília & Centro de Informações Toxicológicas de Brasília \\
& Mato Grosso & Cuiabá & Centro de Informação Antiveneno de Cuiabá \\
& Mato Grosso do & Campo Grande & $\begin{array}{l}\text { Centro Integrado de Vigilância Toxicológica de Cam- } \\
\text { po Grande }\end{array}$ \\
\hline Sul & Paraná & Londrina & Centro de Informações Toxicológicas de Londrina \\
& Rio Grande do Sul & Porto Alegre & Centro de Informação Toxicológica do Rio Grande \\
& Santa Catarina & Florianópolis & Centro de Informação Toxicológica de Santa Catarina \\
\hline Sudeste & Campinas & Centro de Controle de Intoxicações de Campinas \\
& Ribeirão Preto & Centro de Controle de Intoxicações de Ribeirão Preto \\
& São José do Rio & Centro de Assistência Toxicológica de São José do \\
& Preto & Rio Preto \\
& São Paulo & Centro de Assistência Toxicológica do Instituto da \\
& & Criança do Hospital das Clínicas da Faculdade de \\
& & Medicina da Universidade de São Paulo (HCFMUSP) \\
& Centro de Controle de Intoxicações de São Paulo \\
& & Centro de Controle de Intoxicações de Taubaté \\
& & Serviço de Toxicologia de Minas Gerais \\
& &
\end{tabular}

Fonte: Elaboração própria.

*Mantido o nome anterior à publicação da Portaria GM/MS no 1.678, de 2 de outubro de 2015.

\section{Atributos funcionais}

Atendimentos - em maioria, os CIATox oferecem atendimento de forma ininterrupta durante 24 horas/dia, 7 dias/semana e 365 dias/ano ( $\mathrm{n}=18,95 \%)$. Somente um CIATox (5\%) referiu realizar, na atualidade, atendimento de 12 horas diárias em período diurno.

Dos meios de acesso ao atendimento ofertado pelos CIATox (figura 1), cinco (26\%) realizam atendimentos exclusivamente por telefone; quatro (21\%), atendimento telefônico e presencial, em unidades de saúde nas quais o CIATox é vinculado (interconsulta), e três (16\%) oferecem atendimento telefônico e atendimento presencial, tanto em unidades de saúde de vinculação como em sede própria. Um CIATox ( $5 \%$ ), além dos atendimentos telefônico e presencial em unidades de vinculação, referiu oferecer atendimento por meio de redes sociais; e dois (11\%) realizam somente atendimento presencial em unidades de saúde de vinculação. Oito CIATox (42\%) contam com serviço de laboratório para análises toxicológicas; e dois (11\%), para análises clínicas. 
Figura 1. Meios de atendimento oferecidos pelos CIATox por tipo de atendimento. Brasil, 2018

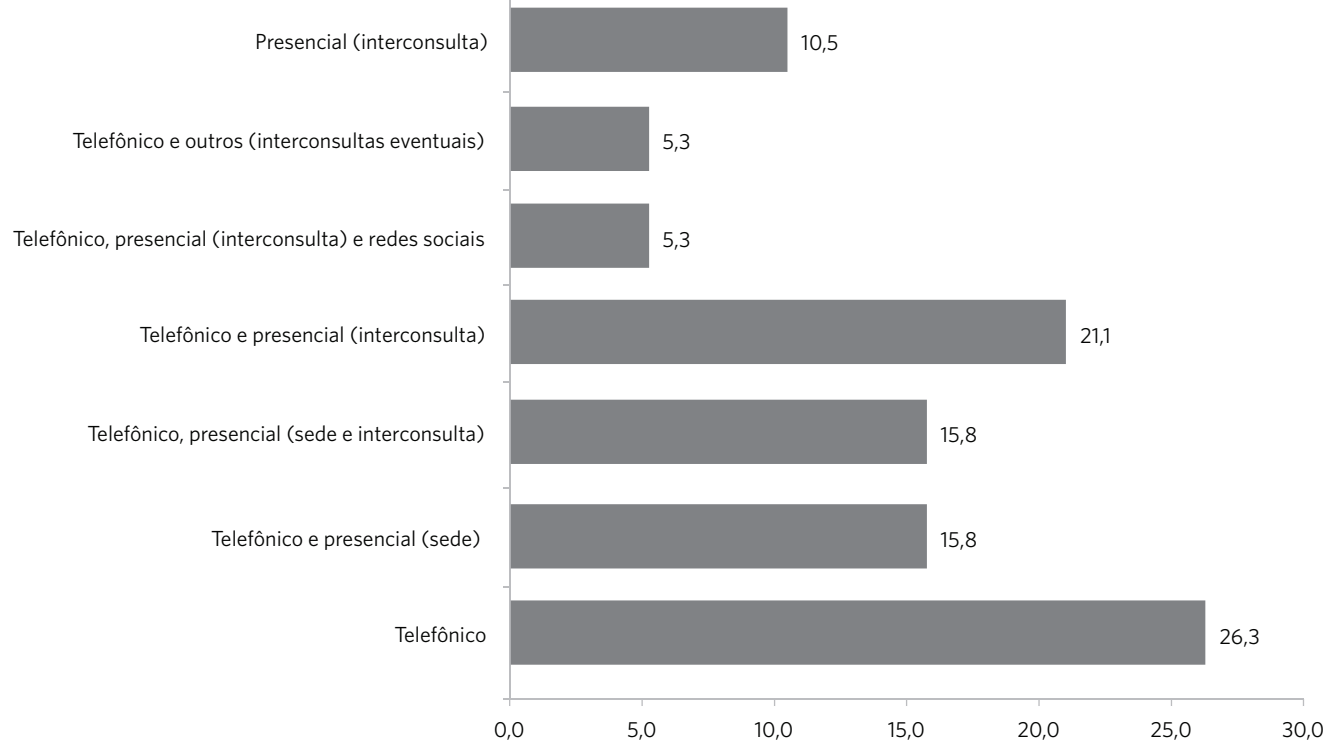

Para 16 CIATox (84\%), o público-alvo de suas atividades são profissionais de saúde e a população em geral. Outrossim, 15 CIATox (79\%) citaram, também, estudantes de graduação e pós-graduação.

Quando perguntados sobre quais instituições ou serviços de saúde são atendidos pelos CIATox, 16 (84\%) referiram como mais frequentes as Unidades Básicas de Saúde, consultórios, unidades de pronto atendimento ou pronto-socorro e os hospitais públicos municipais e estaduais. Em menor frequência $(n=8,42 \%)$, os centros de especialidades e as vigilâncias em saúde em âmbito municipal e estadual.

Atividades ofertadas - ao todo, os CIATox relataram oferecer 39 diferentes atividades (figura 2), variando de 1 a 30, com mediana de 21 atividades. Os 19 CIATox referiram fornecer informação por meio de chamadas telefônicas. Outras atividades destacadas como mais frequentes foram: ensino para graduação $(n=16)$; notificação de eventos toxicológicos $(n=15)$; suporte clínico remoto e suporte clínico presencial a profissionais de saúde (ambos com $\mathrm{n}=14$ ) e registro dos atendimentos em banco de dados de acesso restrito $(\mathrm{n}=14)$. As atividades referidas como menos realizadas foram: ouvidoria, visita domiciliar e consultoria para instituições ou organizações internacionais ( $\mathrm{n}=1, \mathrm{cada})$. 
Figura 2. Atividades ofertadas pelos CIATox. Brasil, 2018

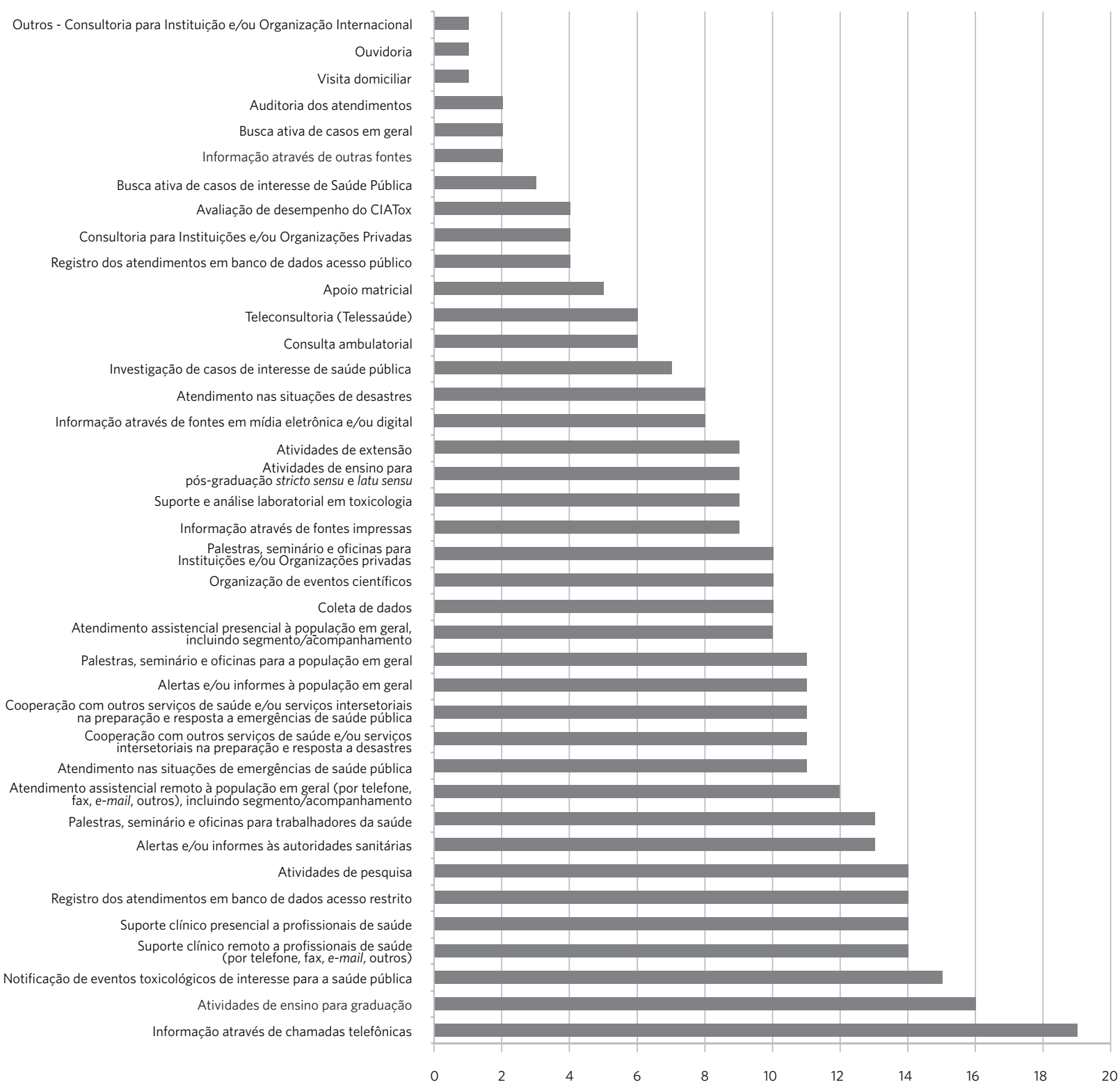

\section{Atributos organizacionais}

Vinculação institucional e gestão - todos os CIATox são vinculados a alguma instituição gestora, dos quais $63 \%(n=12)$ possuem vinculação a uma única instituição; $32 \%(n=6)$, a duas instituições; e $5 \%$ (n=1), a três instituições.

Das instituições de vinculação, nove tipos diferentes foram referidos, considerando tanto as únicas como as múltiplas. São elas: Secretaria de

Saúde Estadual (n=9, 32\%); Secretaria de Saúde Municipal ( $\mathrm{n}=4,14 \%)$; Hospital Universitário Estadual ( $\mathrm{n}=4,14 \%)$; Universidade Estadual ( $\mathrm{n}=3$, $11 \%)$; Universidade Federal ( $\mathrm{n}=2,7 \%)$; Hospital Universitário Federal (n=2,7\%); Hospital Federal ( $n=2,7 \%)$; Hospital Universitário de Fundação Privada, sem fins lucrativos e de caráter filantrópico ( $\mathrm{n}=2,7 \%)$.

As instituições gestoras participam das decisões de coordenação e gerenciamento 
dos CIATox quanto à composição de recursos humanos, materiais e financeiros na maioria dos casos ( $\mathrm{n}=18,95 \%)$, contudo, dez instituições (53\%) não participam das decisões de coordenação e gerenciamento que envolvam a execução das práticas assistenciais promovidas pelos CIATox.

Embora um pouco mais da metade dos CIATox participantes $(\mathrm{n}=10,53 \%$,) tenha referido mudança em suas funções nos últimos dez anos, a estrutura organizacional não sofreu alterações no mesmo período.

Recursos Humanos - A composição de recursos humanos varia conforme o CIATox analisado; contudo, compõem seu quadro de trabalho: profissionais de saúde médicos, enfermeiros, farmacêuticos, psicólogos, assistentes sociais, agentes de saúde, auxiliares e técnicos de laboratório, veterinários e biomédicos; além de profissionais de outras áreas, tais como biólogos, administradores e técnicos em informática.

Destaca-se, ainda, que, em seis CIATox (32\%), parte de seu quadro de profissionais é cedido por outras instituições; e em outros três (16\%), o quadro é composto por voluntários.

Em 14 CIATox (74\%), há algum profissional de saúde graduado presente em todo o horário de funcionamento no serviço. Em se tratando especificamente do profissional médico, cujas atividades sejam direcionadas exclusivamente para as práticas assistenciais, o número reduz para nove (47\%).

Do total de CIATox participantes, 13 (68\%) possuem programa de estágio profissional para o exercício dos plantões diários, direcionado para estudantes de graduação e pós-graduação (aprimoramento e residência), de formação multiprofissional que inclui os cursos de medicina, enfermagem, farmácia, biomedicina e biologia.

Para 17 CIATox (89\%), há dificuldades na composição de recursos humanos decorrente da falta de recursos financeiros e da falta de reposição de profissionais aposentados. Tais dificuldades foram referidas como fator determinante na redução do horário de funcionamento de um dos CIATox de 24 para 12 horas.
Recursos materiais e infraestrutura - 14 CIATox (74\%) dispõem de área física própria; destas, 12 (63\%) são localizadas dentro de um serviço hospitalar. A área é percebida como adequada para 13 CIATox (68\%).

Quanto à disponibilidade de linhas telefônicas para os atendimentos, 17 CIATox (89\%) têm linhas de telefone fixo exclusivo, e cinco (26\%) têm linhas móveis. As linhas fixas disponíveis variam de 1 até 100 ramais dedicados (um único CIATox), com mediana de 2 linhas telefônicas exclusivas por CIATox.

Quanto à disponibilidade de antídotos e soros, 13 CIATox (68\%) não dispõem de estoque de antídotos, e 8 (42\%) não dispõem de estoque de soros antiveneno. O suprimento recebido ou o recurso financeiro para a aquisição desses insumos são provenientes de uma única fonte para 13 CIATox (68\%). Das fontes referidas, seis (32\%) são recursos provenientes exclusivamente da instituição de vinculação; e três (16\%), do governo estadual. Outras fontes citadas foram recursos próprios, instituições ou serviços federais e instituições ou serviços municipais.

Para 13 CIATox (68\%), há dificuldade na composição de recursos materiais e infraestrutura, entre as quais: a aquisição de materiais permanentes, a aquisição de insumos para laboratório, soros e antídotos de alto custo e para a manutenção corretiva e preventiva do espaço físico.

Financiamento - quanto aos recursos financeiros, 17 CIATox (89\%) não possuem financiamento contínuo próprio. Ainda assim, a fonte para 11 CIATox (58\%) é de origem estadual. Três CIATox (16\%) recebem recursos de fonte privada, sendo que a proporção desse recurso é de $99 \%$ para um dos CIATox e de $100 \%$ para outros dois. Três deles (16\%) também obtêm recursos eventuais por prestação de serviços, consultorias ou de agências de fomento à pesquisa.

Dez CIATox (52,63\%) enfrentam dificuldades para obtenção de recursos financeiros. Três CIATox (16\%) não responderam a essa questão. Para os respondentes, os CIATox não possuem autonomia financeira, enfrentam 
limite de cotas mensais das instituições de vinculação e não recebem o incentivo financeiro estabelecido pelo MS.

\section{Discussão}

A Organização Mundial da Saúde preconiza que os países devem ter como ideal ao menos um serviço de informação toxicológica nacional, complementado por Centros satélites regionais, a depender da extensão territorial e populacional10.

No Brasil, atualmente, existe insuficiência e disparidade na cobertura: enquanto sete estados não dispõem de nenhum CIATox (Acre, Roraima, Rondônia, Amapá, Maranhão, Alagoas e Tocantins), um único estado (São Paulo) tem nove CIATox e mais dois serviços públicos que prestam informação toxicológica, mas que não foram incluídos na RUE do SUS (Instituto Butantã, na cidade de São Paulo, e o Centro de Assistência Toxicológica de Presidente Prudente) ${ }^{11,13,21}$. Considerando a disponibilidade e os custos das mais modernas tecnologias de comunicação e as especificidades regionais, seria viável o estabelecimento de uma cobertura mais homogênea no País.

No histórico de criação dos CIATox até a sua inclusão na RUE do SUS, duas iniciativas de origem federal contribuíram para a forma de organização desses serviços. A primeira ocorreu em 1980, quando o MS criou o Sistema Nacional de Informação Tóxico-farmacológicas sob coordenação e administração da Fundação Oswaldo Cruz, que tinha como um dos objetivos ampliar o número de Centros no País por meio de convênios e repasses financeiros entre o MS e as Secretarias Estaduais e Municipais de Saúde, e Fundações de Saúde ligadas às Universidades Públicas. Ainda que tal medida tenha sido preponderante na criação de novos Centros, ela seguia a lógica da organização da assistência à saúde anterior ao SUS, o que explica os diferentes tipos de instituições às quais os CIATox são vinculados, bem como a variedade de atividades por eles desenvolvidas ${ }^{22,23}$.
A segunda iniciativa ocorreu entre $2001 \mathrm{e}$ 2005, quando a então recém-criada Gerência Geral de Toxicologia da Agência Nacional de Vigilância Sanitária promoveu encontros nacionais com os Centros existentes à época, com o objetivo de discutir e viabilizar a atuação da Agência e incrementar a captação dos dados epidemiológicos provenientes dos atendimentos, o que culminou com a criação de uma Rede Nacional de Centros de Informação e Assistência Toxicológica (Renaciat), que estabeleceu critérios e parâmetros para a instalação de novos Centros no País sem, contudo, estabelecer recursos orçamentários para a sua manutenção ${ }^{\mathbf{1 8}}$.

$\mathrm{O}$ fato de as diretrizes para a criação dos primeiros Centros serem anteriores ao SUS pode explicar porque seu desenvolvimento e sua distribuição ocorreram de maneira desigual entre os estados e regiões, bem como as diferenças na organização e nas instituições de vinculação, o que posteriormente se tornou um dos fatores dificultadores para a manutenção da Renaciat ${ }^{\mathbf{1 8}, 23}$.

A ausência de normas de entes federativos quando da constituição dos Centros especializados no atendimento às exposições e intoxicações também ocorreu em países como os Estados Unidos ${ }^{24}$ e o Canadá ${ }^{25}$, sendo que, para este último, a falta de normativa foi considerada como um tipo de 'isolamento' que criou uma barreira para a integração dos Centros com a rede de saúde pública.

Após a inserção dos CIATox na RUE do SUS, as atividades de produção e disseminação de informações; $\mathrm{o}$ atendimento remoto a profissionais de saúde; a notificação dos eventos de interesse em saúde pública; a prevenção de doenças e agravos e promoção da saúde, todos sobre a ocorrência de exposições e intoxicações que interferem no perfil de morbimortalidade da população, foram consideradas como funções essenciais desses serviços. Atividades como busca ativa de casos e investigação de eventos de saúde pública com destaque para exposição e intoxicação; suporte e análise laboratorial toxicológica e a assistência direta à saúde da população nos casos das exposições e 
intoxicações agudas e crônicas foram definidas como funções opcionais dos CIATox ${ }^{\mathbf{1 3}}$.

Todos os CIATox participantes do estudo oferecem atendimento gratuito conjuntamente à rede de serviços de saúde do SUS, a maioria em período contínuo. As diferenças no meio de atendimento se dão pela composição do serviço, e podem ser realizados exclusivamente de forma telefônica (atendimento remoto), ou de maneira presencial, seja em sede própria, seja por meio de interconsultas, como ocorre, por exemplo, nos CIATox vinculados a hospitais que, embora não tenham uma enfermaria ou unidade exclusiva, atendem à demanda por solicitação de outros profissionais da mesma instituição, diretamente nas unidades de pronto atendimento, pronto-socorro ou de internação hospitalar da instituição à qual estão vinculados. Outros CIATox realizam, ainda, análises laboratoriais clínicas e toxicológicas, seja para os atendimentos presenciais, seja como suporte à solicitação de outros serviços de saúde.

Quanto às atividades, todos os CIATox referiram realizar atendimento de informação por meio de chamada telefônica, mesmo os que consideraram seu meio de atendimento como exclusivamente presencial. Marques et al. ${ }^{\mathbf{1 9}}$, em estudo anterior sobre avaliação dos Centros que compunham a Renaciat, observaram que o conceito de 'solicitação de informação' não é claro entre os Centros, o que corrobora um sub-registro de atendimentos. $\mathrm{O}$ atendimento de informação por meio de chamada telefônica corresponde, neste caso, a qualquer solicitação atendida pelos CIATox e que não se constituam de casos de exposição, intoxicação ou envenenamento, nem diagnóstico diferencial.

Quanto às solicitações de atendimento, os CIATox participantes consideraram como público-alvo de suas atividades os profissionais de saúde, estudantes e a população em geral, em proporções semelhantes. Contudo, há uma maior demanda de atendimentos originados de serviços de saúde. Os atendimentos oferecidos pelos CIATox brasileiros diferenciam-se dos atendimentos realizados pelos Centros estadunidenses, nos quais prevalecem as ligações realizadas pelos usuários a partir de suas residências, que recorrem às ligações gratuitas antes de buscarem o atendimento nos serviços pagos de saúde ${ }^{11,26}$.

Para além das atividades essenciais e opcionais designadas, os CIATox participantes do estudo desenvolvem um importante papel na formação de profissionais especializados no atendimento das exposições e intoxicações, por meio da promoção de estágios e atividades de graduação e pós-graduação para estudantes de formação interdisciplinar.

Além da inserção desses profissionais de saúde nos serviços de saúde no âmbito da rede de atenção do SUS, após sua formação e/ou especialização em toxicologia, eles também são requeridos para a composição do quadro de trabalhadores dos próprios CIATox dada a expertise de suas atividades. Nos Centros de países como Suíça e Japão, os profissionais devem passar por treinamentos em serviço ou possuírem pós-graduação em Informação Toxicológica ou Toxicologia Clínica27. Já nos Centros do Canadá e Estados Unidos, os profissionais devem obter uma certificação exclusiva de Especialista em Informação Toxicológica (Specialists in Poison Information) $\mathbf{2 5 , 2 6}$.

Os CIATox participantes referiram autonomia quanto à execução de suas práticas de atenção, contudo, essa autonomia é limitada no que concerne à composição de recursos humanos especializados, aquisição de insumos e recursos materiais necessários ao desenvolvimento de suas atividades. Tais dificuldades foram atribuídas especialmente à falta de financiamento contínuo e adequado que interferem diretamente no funcionamento desses serviços.

Problemas de financiamento são comuns aos Centros internacionais ${ }^{\mathbf{2 8}}$. No Brasil, Bochner ${ }^{23}$ aponta que a dificuldade financeira para a manutenção dos Centros ocorre desde a criação desses serviços, o que culminou com a desativação de três unidades nas cidades de Iguatu (CE) no ano 2000, Rio de Janeiro (RJ) em 2008, Marília (SP) em 2012; e mais recentemente, em 2015, no município Cascavel (PR) ${ }^{29}$. 
Uma possível explicação deve-se à diversidade de órgãos e instituições de vinculação e a percepção dos CIATox apenas como serviços que fornecem informação e/ou promovem assistência somente às intoxicações agudas, fazendo com que os Centros não fossem considerados como serviços integrantes da rede de atenção do SUS e, portanto, não recebessem o financiamento efetivo. Em 2010, com a articulação do Programa Nacional de Telessaúde, do MS, com a Rede Universitária de Telemedicina, do Ministério de Ciência e Tecnologia, que oficializaram no País as ações de teleducação e teleassistência ${ }^{30}$, as funções dos CIATox ganharam relevância, o que favoreceu a integração desses serviços à RUE do SUS, fazendo jus a um incentivo financeiro ${ }^{\mathbf{1 3}}$. Mesmo assim, a questão do financiamento ainda é considerada como ineficiente.

\section{Considerações finais}

Essa caracterização preliminar dos CIATox possui algumas limitações. Em relação às atividades, os respondentes podem subestimar ou superestimar o número e atividades por eles exercidas, o que pode ser entendido como uma das deficiências da ausência de uma diretriz nacional. Além disso, as atividades são condicionadas ao desenvolvimento e funcionamento dos CIATox ao longo do tempo, pelas especificidades regionais, e pelo conhecimento da existência dos CIATox pela população e por outros profissionais de saúde, que podem optar ou não pela consulta ao Centro. Ainda que o estudo não tenha sido realizado com a totalidade dos CIATox brasileiros, os resultados apresentados permitem inferências no entendimento de como se dá a organização dos CIATox não participantes, uma vez que esses Centros também seguem as mesmas instruções quanto ao exercício das funções essenciais e opcionais e quanto à sua inserção na RUE no âmbito do SUS.

As exposições e intoxicações representam problemas de saúde pública que necessitam ser reconhecidos pelo setor saúde. Atualmente, tramita na Câmara dos Deputados o Projeto de Lei (PL) no 9.006/2017 (anteriormente designado como PL 96/2014) que propõe a alteração da Lei $n^{0} 8.080 / 90$ para incluir no âmbito do SUS uma política de informação e assistência toxicológica e de logística de antídotos $^{31}$. Nesse aspecto, os CIATox podem contribuir com sua expertise para a visibilidade das exposições e intoxicações no Brasil a partir do desenvolvimento de práticas de atenção que incluem a promoção, prevenção, diagnóstico e tratamento à pessoa exposta ou intoxicada. Tais práticas, contudo, devem ser integradas e operacionalizadas em uma rede de atenção que inclua ainda outros serviços de saúde distribuídos no território e organizados nos diferentes níveis de atenção.

\section{Colaboradores}

Costa AO (0000-0001-7336-8795)* contribuiu para a concepção e planejamento do estudo; análise e interpretação dos dados; elaboração do rascunho; e aprovação final do manuscrito. Alonzo HGA (0000-0002-3366-0983)* contribuiu para a concepção e planejamento do estudo; análise e interpretação dos dados; revisão crítica do conteúdo; e aprovação da versão final do manuscrito. 


\section{Referências}

1. World Health Organization. The public health impact of chemicals: knows and unknows [internet]. Geneva, Switzerland: WHO; 2016 [acesso em 2018 maio 23]. Disponível em: http://apps.who.int/iris/ bitstream/handle/10665/206553/WHO_FWC_PHE_ EPE_16.01_eng.pdf?sequence=1.

2. World Health Organization. The global burden of disease: 2004 update. Geneva, Switzerland: WHO; 2008.

3. World Health Organization. Mortality due to unintentional poisoning. World Health Statistics: 2016 Monitoring Health for the SDGs [internet]. Geneva: WHO; 2016 [acesso em 2018 maio 29]. Disponível em: http://www.who.int/gho/publications/world_ health_statistics/2016/whs2016_AnnexA_MortalityPoisoning.pdf?ua=1\&ua=1.

4. World Health Organization. World health statistics 2018: monitoring health for the SDGs, sustainable development goals [internet]. Geneva: WHO; 2018 [acesso em 2018 maio 23]. Disponível em: http://apps.who.int/iris/ bitstream/handle/10665/272596/9789241565585-eng. pdf?ua=1\&ua=1.

5. World Health Organization. Global Health Estimates 2016: Deaths by cause, age, sex, by country and by region, 2000-2016 [internet]. Geneva, Switzerland; WHO; 2018 [acesso em 2018 maio 23]. Disponível em: http://www.who.int/healthinfo/global_burden_disease/estimates/en/.

6. Brasil. Ministério da Saúde. Secretaria de Vigilância em Saúde, Coordenação-Geral de Desenvolvimento da Epidemiologia em Serviços. Guia de Vigilância em Saúde. 2. ed. Brasília, DF: MS; 2017.

7. Brasil. Ministério da Saúde. Departamento de Informática do SUS. TabNet Win32 3.0: Intoxicação Exógena - Notificações registradas no Sinan Net - Brasil [internet]. 2018 [acesso em 2018 maio 23]. Disponível em: http://tabnet.datasus.gov.br/cgi/deftohtm. exe?sinannet/cnv/Intoxbr.def.

8. Albuquerque PCC, Gurgel IGD, Gurgel AM, et al. He- alth information systems and pesticide poisoning at Pernambuco. Rev. bras. epidemiol. [internet]. 2015 jul-set [acesso em 2018 maio 23]; 18(3):666-678. Disponível em: http://www.scielo.br/scielo.php?script=sci arttext\&pid=S1415-790X2015000300666.

9. Germano LC, Alonzo HGA. Intoxicações e reações adversas a medicamentos: perfil local de subnotificação aos sistemas de informação em saúde. Rev. Eletrônica de Farmácia [internet]. $2015 \mathrm{dez}$ [acesso em 2018 maio 23]; 12(4). Disponível em: https://revistas. ufg.br/REF/article/view/36725.

10. Organización Mundial de la Salud, Programa Internacional de Seguridad de las Substancias Químicas. Directrices para la lucha contra las intoxicaciones. Genebra: OMS; 1998.

11. Costa AO. Atendimentos registrados no Centro de Controle de Intoxicações de Campinas: análise do período de 1998 a 2011 [dissertação] [internet]. Campinas: Unicamp; 2015 [acesso em 2018 maio 14]. Disponível em: http://repositorio.unicamp.br/jspui/handle/REPOSIP/312705.

12. Baroud R. Concepção e organização de um centro de controle de intoxicações. Rev. Saúde Públ. 1985;19(6):556-565.

13. Brasil. Ministério da Saúde. Portaria GM/MS no 1.678, de 2 de outubro de 2015. Institui os Centros de Informação e Assistência Toxicológica (CIATox) como estabelecimentos de saúde integrantes da Linha de Cuidado ao Trauma, da Rede de Atenção as Urgências e Emergências no âmbito do Sistema Único de Saúde - SUS. Diário Oficial da União. 6 Dez 2015. [acesso em 2019 fev 12]. Disponível em: http://bvsms.saude.gov.br/bvs/saudelegis/gm/2015/prt1678_02_10_2015.html.

14. Alonzo HGA. Consultas em seis centros de controle de intoxicações do Brasil: análise dos casos, hospitalizações e óbitos [tese] [internet]. Campinas: Universidade Estadual de Campinas; 2010. [acesso em 2018 maio 23]. Disponível em: http://repositorio.unicamp. br/jspui/handle/REPOSIP/309357. 
15. Bernardes SS, Turini CA, Matsuo T. Profile of suicide attempts using intentional overdose with medicines, treated by a poison control center in Paraná State, Brazil. Cad. Saúde Pública. 2010; 26(7):1366-1372.

16. Costa AO, Alonzo HGA. Casos de exposições e Intoxicações por medicamentos registrados em um Centro de Controle de intoxicações do interior do Estado de São Paulo. Rev. Bras. Pesq. Saúde [internet]. 2015 abr/jun [acesso em 2018 maio 18]; 17(2):52-60. Disponível em: http://periodicos.ufes.br/RBPS/article/ download/13188/9238.

17. Presgrave RF, Camacho LAB, Villas Boas MHS. A profile of unintentional poisoning caused by household cleaning products, disinfectants and pesticides. Cad. Saúde Pública. 2008; 24(12):2901-2908.

18. Azevedo JLS. A importância dos Centros de Informação e Assistência Toxicológica e sua contribuição na minimização dos agravos à saúde e ao meio ambiente no Brasil [dissertação] [internet]. Brasília, DF: Universidade de Brasília; 2006. 247 p. [acesso em 2018 maio 23]. Disponível em: http://repositorio.unb.br/ bitstream/10482/4740/1/2006_Jorge\%20Luiz\%20 Sayde\%20de\%20Azevedo.pdf.

19. Marques MB, Bortoletto ME, Bezerra MCC, et al. Avaliação da rede brasileira de Centros de Controle de Intoxicações a Envenenamento - CCIEs. Cad. Saúde Pública [internet]. 1995 out/dez [acesso em 2018 maio 15]; 11(4):560-578. Disponível em: http:// http://www.scielo.br/pdf/csp/vlln4/vlln4a04.pdf.

20. Joomla!TM. Version 3.9, Open Source; c2005-2018 [internet]. [acesso em 2018 fev 15]. Disponível em: https://www.joomla.org/.

21. World Health Organization. World directory of Poison Control Centers [internet]. Geneva: WHO; 2017 [acesso em 2018 jun 13]. Disponível em: http://www.who.int/ gho/phe/chemical_safety/poisons_centres/en/.

22. Gandolfi E. Estudo epidemiológico dos eventos toxicológicos relacionados a medicamentos no Estado de São Paulo [dissertação]. [Campinas]: Universidade Estadual de Campinas; 2002. 335 p.
23. Bochner R. Informação sobre intoxicações e envenenamentos para a gestão do SUS: um panorama do Sistema Nacional de Informações Tóxico-Farmacológicas - Sinitox. RECIIS [internet]. 2013 jun [acesso em 2018 maio 10]; 7(2). Disponível em: http:// www.reciis.icict.fiocruz.br/index.php/reciis/article/view/472/1123.

24. Institute of Medicine (US), Committee on Poison Prevention and Control. Forging a Poison Prevention and Control System. Washington-DC: National Academies Press; 2004.

25. Durigon M, Elliott C, Purssell R, et al. Canadian Poison Control Centres: preliminary assessment of their potential as a resource for public health surveillance. Clin Toxicol (Phila). 2013; 51(9):886-891.

26. American Association of Poison Control Centers [internet]. Alexandria: AAPCC; c2017 [acesso em 2018 maio 26]. Disponível em: http://www.aapcc.org/.

27. Pourmand A, Wang J, Mazer M. A survey of poison control centers worldwide. Daru. 2012; 20(1):13.

28. Thompson CA. Poison Control Centers suffer state budget cuts. Am J health-system Pharmacy. 2009; 66(19):1682-1687.

29. Universidade Estadual do Oeste do Paraná. Ofício n ${ }^{\circ}$. 15/2015. Ceatox: Cascavel; 2015. [Circular Interna].

30. Silva AB, Moraes IHS. O caso da Rede Universitária de Telemedicina: análise da entrada da telessaúde na agenda política brasileira. Physis. 2012; 22(3):1211-1235.

31. Brasil. Câmara dos Deputados. Projeto de Lei - PL 9006/2017 [internet]. 2017 [acesso em 2018 jun 15]. Disponivel em: http://www.camara.gov.br/proposicoesWeb/fichadetramitacao?idProposicao=2160132.

Recebido em 08/08/2018

Aprovado em 16/12/2018

Conflito de interesses: inexistente

Suporte financeiro: não houve 